\title{
Universal quantum computing with semiconductor double-dot molecules on a chip
}

\author{
Peng Xue \\ Department of Physics, Southeast University, Nanjing 211189, P. R. China and \\ Institute for Quantum Information Science, University of Calgary, Alberta T2N 1N4, Canada
}

(Dated: November 3, 2018)

\begin{abstract}
We develop a scalable architecture for quantum computation using controllable electrons of double-dot molecules coupled to a microwave stripline resonator on a chip, which satisfies all Divincenzo criteria. We analyze the performance and stability of all required operations and emphasize that all techniques are feasible with current experimental technologies.
\end{abstract}

PACS numbers: 03.67.Lx, 42.50.Pq, 73.21.La

\section{INTRODUCTION}

Quantum computing enables some computational problems to be solved faster than would ever be possible with a classical computer [1] and exponentially speeds up solutions to other problems over the best known classical algorithms [2]. Of the promising technologies for quantum computing, solid-state implementations such as spin qubits in quantum dots 3 and bulk silicon [4] , and charge qubits in bulk silicon [5] and in superconducting Josephson junctions [6], are especially attractive because of stability and expected scalability of solid-state systems; of these competing technologies, semiconductor double-dot molecules (DDMs) are particularly important because of the combination spin and charge manipulation to take advantage of long memory times associated with spin states and at the same time to enable efficient readout and coherent manipulation of charge states.

Here we develop a scalable architecture for semiconductor quantum computation based on two-electron states in DDMs [7 11] coupled to a microwave stripline resonator [12, 13]. The quantum information is encoded in the superpositions of double-dot singlet states. The initialization of qubit states can be implemented by an adiabatic passage. A universal set of gates including single- and two-qubit gates can be implemented via the resonator-assisted interaction with a microwave stripline resonator and the requirement for electrically driving DDMs directly is released, which avoids moving the system away from the optimal point (where the coupling is achieved to be strongest) because of the potential difference caused by the electric drive and increasing the extended dephasing due to the fluctuations of the electric field. Compared to the previous protocol [13], the system in our scheme always works in the strong coupling regime and the second order dephasing time is considered here. The readout of qubits can be realized via microwave irradiation of the resonator by probing the transmitted or reflected photons. The main decoherence processes are dissipation of the stripline resonator, charge-based relaxation and dephasing of the semiconductor DDMs, and spin dephasing limited by hyperfine interactions with nuclei. By numerical analysis we show all gate operations and measurements can be implemented within the coher- (a) $\left|T_{-}\right\rangle$
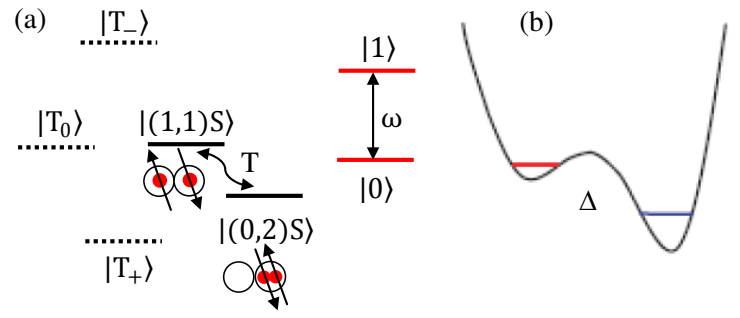

FIG. 1: (a) Energy level diagram showing the $(0,2)$ and $(1,1)$ singlets in solid lines and the three $(1,1)$ triplets in dashed lines, and the logical qubit states $|0\rangle$ and $|1\rangle$ in red lines. (b) Schematic of the double-well potential with an energy offset $\Delta$ provided by the external electric field along $x$ axis.

ent life time of qubits. Thus we address all Divincenzo criteria [14] and show all play important roles in the dynamics of the two-electron system but none represents a fundamental limit for quantum computing.

\section{QUBITS}

We consider the system with two electrons located in adjacent quantum dots coupling via tunneling. Imagine one of the dots is capacitively coupled to a stripline resonator 10, 12, 13]. With an external magnetic field $B_{z}=100 \mathrm{mT}$ along $z$ axis, the spin aligned states $\left|T_{+}\right\rangle=$ $|\uparrow \uparrow\rangle$ and $\left|T_{-}\right\rangle=|\downarrow \downarrow\rangle$, and the spin-anti-aligned states $\left|T_{0}\right\rangle=(|\downarrow \uparrow\rangle+|\downarrow \uparrow\rangle) / \sqrt{2}$ and $|(1,1) S\rangle=(|\downarrow \uparrow\rangle-|\downarrow \uparrow\rangle) / \sqrt{2}$ have energy gaps due to the Zeeman splitting shown in Fig. 1(a). The notation $\left(n_{L}, n_{R}\right)$ labels the number of electrons in the left and right quantum dots. The doubly occupied state $|(0,2) S\rangle$ is coupled via tunneling $T$ to the singlet state $|(1,1) S\rangle$. The double-dot system can be described by an extended Hubbard Hamiltonian $\hat{H}=\left(E_{\mathrm{os}}+\mu\right) \sum_{i, \sigma} \hat{n}_{i, \sigma}-T \sum_{\sigma}\left(\hat{c}_{L, \sigma}^{\dagger} \hat{c}_{R, \sigma}+\mathrm{hc}\right)+$ $U \sum_{i} \hat{n}_{i, \uparrow} \hat{n}_{i, \downarrow}+W \sum_{\sigma, \sigma^{\prime}} \hat{n}_{L, \sigma} \hat{n}_{R, \sigma^{\prime}}+\Delta \sum_{\sigma}\left(\hat{n}_{L, \sigma}-\hat{n}_{R, \sigma}\right)$ for $\hat{c}_{i, \sigma}\left(\hat{c}_{i, \sigma}^{\dagger}\right)$ annihilating (creating) an electron in quantum dot $i \in\{L, R\}$ with spin $\sigma \in\{\uparrow, \downarrow\}, \hat{n}_{i, \sigma}=\hat{c}_{i, \sigma}^{\dagger} \hat{c}_{i, \sigma}$ a number operator, and $\Delta$ an energy offset yielded by the external electric field along $x$ axis shown in Fig. 1(b). The first term corresponds to on-site energy $E_{\mathrm{os}}$ plus 
site-dependent field-induced corrections $\mu$. The second term accounts for $i \leftrightarrow j$ electron tunneling with rate $T$, and the third term is the on-site charging cost $U$ to put two electrons with opposite spin in the same dot, and the fourth term corresponds to inter-site Coulomb repulsion. In the basis $\{|(1,1) S\rangle,|(0,2) S\rangle\}$, the Hamiltonian can be deduced as

$$
\hat{H}_{\mathrm{d}}=-\Delta|(0,2) S\rangle\langle(0,2) S|+T|(1,1) S\rangle\langle(0,2) S|+\text { hc. }
$$

With the energy offset $\Delta$, degenerate perturbation theory in the tunneling $T$ reveals an avoided crossing at this balanced point between $|(1,1) S\rangle$ and $|(0,2) S\rangle$ with an energy gap $\omega=\sqrt{\Delta^{2}+4 T^{2}}$, and the effective tunneling between the left and right dots with the biased energies $\Delta$ is changed from $T$ to $T^{\prime}=\omega / 2$. We choose the superpositions of the singlet states as our qubit states:

$$
\begin{aligned}
|0\rangle & \equiv(|(1,1) S\rangle-|(0,2) S\rangle) / \sqrt{2} \\
|1\rangle & \equiv(|(1,1) S\rangle+|(0,2) S\rangle) / \sqrt{2} .
\end{aligned}
$$

The essential idea is to use an effective electric dipole moment of the singlet states $|(1,1) S\rangle$ and $|(0,2) S\rangle$ of a DDM coupled to the oscillating voltage associated with a stripline resonator shown in Fig. 2(a). We consider a stripline resonator with length $L$, the capacitance coupling of the resonator to the $\operatorname{dot} C_{\mathrm{c}}$, the capacitance per unit length $C_{0}$, the total capacitance of the double-dot $C_{\text {tot }}$, and characteristic impedance $Z_{0}$. The fundamental mode frequency of the resonator is $\omega_{0}=\pi / L Z_{0} C_{0}$. The resonator is coupled to a capacitor $C_{e}$ for writing and reading the signals. Neglecting the higher modes of the resonator and working in the rotating frame with the rotating wave approximation, we obtain an effective interaction Hamiltonian as

$$
\hat{H}_{\mathrm{int}}=g\left(\hat{a} \hat{\sigma}_{+}+\mathrm{hc}\right)
$$

with $\hat{a}\left(\hat{a}^{\dagger}\right)$ the annihilation (creation) operator of the resonator field, $\hat{\sigma}_{+}=|1\rangle\left\langle 0\left|, \hat{\sigma}_{-}=\right| 0\right\rangle\langle 1|$, and the effective coupling coefficient

$$
g=\frac{1}{2} e \frac{C_{\mathrm{c}}}{L C_{\mathrm{tot}} C_{0}} \sqrt{\frac{\pi}{Z_{0}}} \sin 2 \theta
$$

with $\theta=\frac{1}{2} \tan ^{-1}\left(\frac{2 T}{\Delta}\right)$.

The interaction between the resonator and qubit states is switchable via tuning the electric field along $x$ axis. In the case of the energy offset yielded by the electric field $\Delta \approx 0$, we obtain the maximum value of the coupling between the resonator and singlets in double dots. That is so called the optimal point. Whereas $\Delta \gg T, \theta$ tends to 0 , the interaction is switched off.

\section{INITIALIZATION AND TRANSPORTATION}

Initialization of qubit states can be implemented by an adiabatic passage between the two singlet states [11].

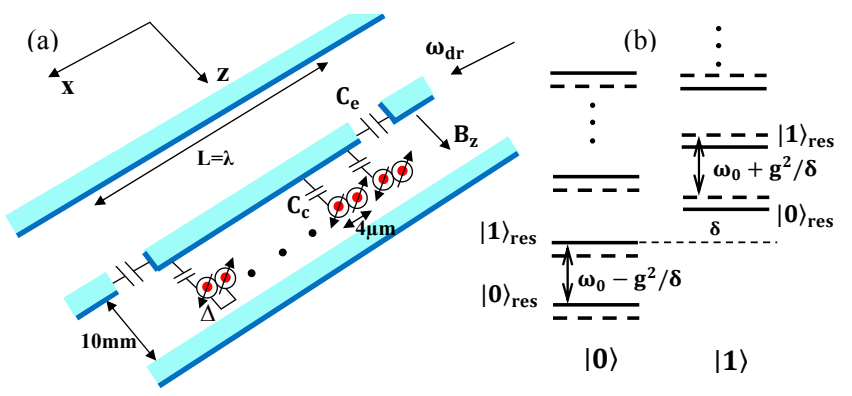

FIG. 2: (a) Schematic of DDMs, biased with an energy offset $\Delta$, capacitively coupled to the stripline resonator. The coupling can be switched on and off via the external electric field along $x$ axis. The stripline resonator is driven by a classical field along $x$ axis. (b) Energy spectrum of the bared (solid lines) and dressed (dashed lines) states in the dispersive regime.

Controllably changing $\Delta$ allows for adiabatic passage to past the charge transition, with $|(0,2) S\rangle$ as the ground state if $\Delta \gg T$ achieved. First we turn on the external electric field along $x$ axis and prepare the two electrons of DDMs in the state $|(0,2) S\rangle$ by a large energy offset $\Delta$. We change $\vartheta$ adiabatically to $\pi / 4$ by tuning the electric field, and then initialize the qubits in the qubit state $|0\rangle$.

The SWAP operation [12], where a qubit state is swapped with a photonic state of the resonator, can be used to implement transmission of qubits. If there is no photon in the resonator, with the detuning $\delta=\left|\omega-\omega_{0}\right|=$ 0 and evolution time $\pi / g$, a qubit is mapped to the photonic state in the resonator $(\alpha|0\rangle+\beta|1\rangle)|0\rangle_{\text {res }} \longrightarrow$ $|0\rangle(\alpha|0\rangle+\beta|1\rangle)_{\text {res }}$. Then we switch off the coupling between this qubit and resonator and switch on that between the desired qubit and resonator via the local electric fields along $x$ axis. After the same evolution time, the previous qubit state is transmitted to the desired qubit via the interaction with the resonator.

\section{A UNIVERSAL SET OF GATES}

Single-qubit gates including bit-flip and phase gates, and an entangling two-qubit gate can be implemented via resonator-associated interaction with a stripline resonator. We consider a DDM interacts with a stripline resonator field, which is driven by a strong classical field along $x$ axis

$$
\hat{H}_{\mathrm{dr}}(t)=\Omega\left(\hat{a}^{\dagger} e^{-\mathrm{i} \omega_{\mathrm{dr}} t}+\mathrm{hc}\right)
$$

with the Rabi frequency $\Omega$, and the frequency of the classical field $\omega_{\mathrm{dr}}$ substantially detuned from the resonator frequency $\omega_{0}$. In the rotating frame at the frequency $\omega_{\mathrm{dr}}$ for a single qubit and the field, we obtain

$$
\hat{H}_{1 q}=\left(\omega_{0}-\omega_{\mathrm{dr}}\right) \hat{a}^{\dagger} \hat{a}+\frac{\omega-\omega_{\mathrm{dr}}}{2} \hat{\sigma}_{z}-g\left(\hat{a}^{\dagger} \sigma_{-}+\mathrm{hc}\right)+\frac{\Omega_{\mathrm{R}}}{2} \hat{\sigma}_{x}
$$


with the effective Rabi frequency $\Omega_{\mathrm{R}}=\frac{2 \Omega g}{\omega_{0}-\omega_{\mathrm{gr}}}$.

In the dispersive regime $\delta \gg g$, we obtain the effective Hamiltonian from Eq. (6) as

$$
\hat{H}_{x}=\left(\omega_{0}-\omega_{\mathrm{dr}}\right) \hat{a}^{\dagger} \hat{a}+\frac{\omega+g^{2} / \delta-\omega_{\mathrm{dr}}}{2} \hat{\sigma}_{z}+\frac{\Omega_{\mathrm{R}}}{2} \hat{\sigma}_{x} .
$$

By choosing $\omega_{\mathrm{dr}}=\omega+g^{2} / \delta$, the Hamiltonian (7) evolves as a rotation around the $x$ axis. The gate operates on the time scaling $t_{x} \sim 1 / \Omega_{\mathrm{R}}$. These Rabi oscillations have already been observed experimentally in [6] .

In another case the drive is sufficiently detuned from the qubit $\left|\omega-\omega_{\mathrm{dr}}\right| \gg \Omega_{\mathrm{R}}$ [15], we obtain a different effective Hamiltonian from Eq. (6)

$$
\hat{H}_{z}=\left(\omega_{0}-\omega_{\mathrm{dr}}\right) \hat{a}^{\dagger} \hat{a}+\frac{\Omega_{\mathrm{R}}^{\prime}}{2} \hat{\sigma}_{z},
$$

which generates rotations around $z$ axis at a rate $\Omega_{\mathrm{R}}^{\prime}=$ $\omega+g^{2} / \delta-\omega_{\mathrm{dr}}+\frac{1}{2} \frac{\Omega_{\mathrm{R}}^{2}}{\omega-\omega_{\mathrm{dr}}}$. The time scaling of this gate operation is $t_{z} \sim 1 / \Omega_{\mathrm{R}}^{\prime}$.

Since we can switch on and off the coupling between the resonator and any DDM by tuning the local electric fields along $x$ axis, for the case of two identical DDMs simultaneously coupled to the resonator, without the drive, in the dispersive regime, we obtain the effective Hamiltonian for the system from Eq. (3)

$$
\begin{aligned}
\hat{H}_{2 q}= & \left(\omega_{0}+\frac{g^{2}}{\delta} \sum_{i=1,2} \hat{\sigma}_{z}^{i}\right) \hat{a}^{\dagger} \hat{a}+\frac{1}{2}\left(\omega+\frac{g^{2}}{\delta}\right) \sum_{i=1,2} \hat{\sigma}_{z}^{i} \\
& +\frac{g^{2}}{\delta}\left(\hat{\sigma}_{+}^{1} \hat{\sigma}_{-}^{2}+\mathrm{hc}\right) .
\end{aligned}
$$

In the rotating frame at the frequency $\omega$, the evolution operation of the two-qubit system dominated by the above Hamiltonian after tracing out the field state (assume there is one photon in the resonator)

$$
U_{2 q}=\exp \left\{-\mathrm{i} \frac{g^{2}}{\delta} t \frac{3}{2} \sum_{i=1,2} \hat{\sigma}_{z}^{i}\right\} \sqrt{\mathrm{iSWAP}}
$$

which provides an entangling two-qubit gate - root of SWAP gate on a time scaling $t_{2 q}$ that satisfies $g^{2} t_{2 q} / \delta=$ $\pi / 4$.

Hence we have built a universal set of gates for quantum computing with semiconductor DDMs coupled to a stripline resonator field. Compared to the previous protocols [13, 16], we drive the resonator instead of driving qubits directly to implement single-qubit gates, in which no addressing qubits individually is required. The feasibility of single-qubit gates has already been proved in 6] experimentally. For the two-qubit gate, we realize it with the off-resonant interaction between both qubits and resonator and release the requirement for driving qubits with a strong classical field [13, 16]. Compared to the previous protocol [13], the system works in the strong coupling regime and the second order dephasing time is considered. Driving qubits introduces large energy difference between the potentials which moves the system away from the optimal point as presented in [13]. In that case the interaction is out of the strong coupling regime even with the maximum coupling, e.g. $g T_{2}<1$ with $T_{2}$ the extended dephasing time.

\section{READOUT}

To perform a measurement of qubits, a drive of frequency $\omega_{\mathrm{dr}}$ modeled by Eq. (5) is sent through the resonator. In the dispersive regime, the energy gap between the dressed states $|0\rangle_{\text {res }}$ and $|1\rangle_{\text {res }}$ is $\omega_{0}-g^{2} / \delta$ for the qubit in the state $|0\rangle$, while the energy gap $\omega_{0}+g^{2} / \delta$ for the state $|1\rangle$ shown in Fig. 2(b). The matrix element of the Hamiltonian $\hat{H}_{\mathrm{dr}}$ corresponding to a bit-flip from the state $|1\rangle$ is suppressed, and depending on the qubit being in the states $|0\rangle$ or $|1\rangle$ the transmission spectrum will present a peak of width $\kappa$ (the resonator decay rate) at $\omega_{0}-g^{2} / \delta$ or $\omega_{0}+g^{2} / \delta$. This dispersive pull of the resonator frequency is $\pm g^{2} / \kappa \delta$, and the pull is power dependent and decreases in magnitude for photon numbers inside the resonator [17]. Via microwave irradiation of the resonator by probing the transmitted or reflected photons, the readout of qubits can be realized and completed on a time scaling $t_{m}=1 / \gamma_{\phi}$, where $\gamma_{\phi}=8 \bar{n}\left(\frac{g^{2}}{\delta}\right)^{2} \frac{1}{\kappa}$ is the dephasing rate due to quantum fluctuations of the number of photon $\bar{n}$ within the resonator.

\section{DECOHERENCE}

Now we analyze the dominant noise source of our system including the charge-based dephasing and relaxation, the spin phase noise due to hyperfine coupling and the photon loss. Coupling to a phonon bath causes relaxation of the charge system in a time $T_{1}$. The characteristic charge dephasing with a rate $T_{2}^{-1}$. The time-ensembleaveraged dephasing time $T_{2}^{*}$ is limited by hyperfine interactions with nuclear spins. The decay of the resonator $\kappa$ is considered as another dominant source of decoherence.

For the charge relaxation time $T_{1}$, the decay is caused by coupling qubits to a phonon bath. With the spinboson model, the perturbation theory gives an overall error rate from the relaxation and incoherent excitation, with which one can estimate the relaxation time $T_{1} \sim$ $1 \mu \mathrm{s}$ [12].

The charge dephasing $T_{2}$ rises from variations of the energy offset $\delta(t)=\delta+\epsilon(t)$ with $\left\langle\epsilon(t) \epsilon\left(t^{\prime}\right)\right\rangle=$ $\int d \omega S(\omega) e^{i \omega\left(t-t^{\prime}\right)}$, which is caused by the low frequency fluctuation of the electric field. The gate bias of the qubit drifts randomly when an electron tunnels between the metallic electrode. Due to the low frequency property, the effect of the $1 / f$ noise on the qubit is dephasing rather than relaxation. At the zero derivative point, compared to a bare dephasing time $T_{b}=1 / \sqrt{\int d \omega S(\omega)}$, the charge dephasing is $T_{2} \sim \omega T_{b}^{2}$ near the optimal point $\delta=0$. The bare dephasing time $T_{b} \sim 1$ ns was observed in [18]. Then 
the charge dephasing is estimated as $T_{2} \sim 10-100 \mathrm{~ns}$. Using quantum control techniques, such as better highand low-frequency filtering of electronic noise, $T_{b}$ exceeding $1 \mu \mathrm{s}$ was observed [8], which suppresses the charge dephasing.

The hyperfine interactions with the gallium arsenide host nuclei causes nuclear spin-related dephasing $T_{2}^{*}$. The hyperfine field can be treated as a static quantity, because the evolution of the random hyperfine field is several orders slower than the electron spin dephasing. In the operating point, the most important decoherence due to hyperfine field is the dephasing between the singlet state $|(1,1) S\rangle$ and one of the triplet state $\left|T_{0}\right\rangle$. By suppressing nuclear spin fluctuation, the dephasing time can be obtained by quasi-static approximation as $T_{2}^{*}=1 / g \mu_{B}\left\langle\Delta B_{n}^{z}\right\rangle_{\mathrm{rms}}$, where $\Delta B_{n}^{z}$ is the nuclear hyperfine gradient field between two coupled dots and rms means a root-mean-square time-ensemble average. A measurement of the dephasing time $T_{2}^{*} \sim 10 \mathrm{~ns}$ was demonstrated in 8].

The quality factor $Q$ of the superconducting resonator in the microwave domain can be achieved $10^{6}$ [19]. In practice, the local external magnetic field $\sim 100 \mathrm{mT}$ reduces the limit of the quality factor to $Q \sim 10^{4}[20]$. The dissipation of the resonator $\kappa=\omega_{0} / Q$ leads the decay time about $1 \mu \mathrm{s}$ with the parameters $\omega_{0}=2 \pi \times 10 \mathrm{GHz}$.

\section{FEASIBILITY}

Now we analyze the feasibility of the proposal with a gate-defined double-dot device as an example which is fabricated using a GaAs/AlGaAs heterostructure grown by molecular beam epitaxy with a two-dimensional electron gas 100nm below the surface, with density $2 \times$ $10^{11} \mathrm{~cm}^{2}$ [8]. When biased with negative voltages, the patterned gates create a double-well potential shown in Fig. 1(b). The quantum-mechanical tunneling $T$ between the two quantum dots is about $T \simeq 0-10 \mu \mathrm{eV}$. The stripline resonator can be fabricated with existing lithography techniques [19]. The qubit can be placed within the resonator formed by the transmission line to strongly suppress the spontaneous emission. The stripline resonator in coplanar waveguides with $Q \sim 10^{4}$ have already been demonstrated in 20]. The diameter of the quantum dot is about $400 \mathrm{~nm}$, and the corresponding capacitance of the double-dot $C_{\text {tot }}$ is about $200 \mathrm{aF}$. The capacitive coupling of the resonator to the dot is about $C_{\mathrm{c}} \approx 2 C_{\mathrm{tot}}=400 \mathrm{aF}$. In practice, for $\omega_{0}=2 \pi \times 10 \mathrm{GHz}$, $Z_{0}=50 \Omega, L \sim \lambda=3 \mathrm{~cm}$, the coupling coefficient $g \sim 2 \pi \times 125 \mathrm{MHz}$ is achievable by the numerical estimations in 4 . The frequency and coupling coefficient can be tuned by changing $L C_{0}$. The external magnetic field along $z$ axis is about $B_{z}=100 \mathrm{mT}$ to make sure the energy splitting $E_{z}=g \mu_{B} B_{z}$ between the two triplet states $\left|T_{ \pm}\right\rangle$is larger than $\omega \sim \omega_{0}$.

With these parameters we can estimate the time scaling for quantum computing. The time for transmit- ting a qubit to a photonic qubit in the resonator is about $t_{\mathrm{tr}}=\pi / g \approx 4 \mathrm{~ns}$. Readout of qubits takes the time $t_{m} \approx 0.02 \mathrm{~ns}$ in the case $\bar{n}=1$ with the parameters $\left\{\omega_{0}, \omega, \omega_{\mathrm{dr}}, g, \Omega\right\} / 2 \pi=\{10,5,5,0.125,10\} \mathrm{GHz}$. The operating time for the single-qubit rotation along $x$ axis is $t_{x} \sim 1 / \Omega_{\mathrm{R}} \approx 0.3 \mathrm{~ns}$ with the above parameters. The single-qubit rotation along $z$ axis takes a time $t_{z} \sim 1 / \Omega_{\mathrm{R}}^{\prime} \approx 0.03 \mathrm{~ns}$ with the parameters different from above to obtain the desired evolution of the system, that is $\omega / 2 \pi \approx 1 \mathrm{MHz}$ (the rest are same). The two-qubit gate in (9) can be realized on the time scaling $t_{2 q}$ which satisfies $g^{2} t_{2 q} / \delta=\pi / 4$ and is calculated as $t_{2 q} \approx 8$ ns with the parameters $\{g, \delta\} / 2 \pi=\{0.125,1\} \mathrm{GHz}$. Here for the twoqubit gate we choose the tunneling $T \approx 18 \mu \mathrm{eV}$ which was recently realized in 21]. Thus, all these operating times are less than the minimum decoherence time.

Now we analyze the effect on gate operations due to noise. The variations of the energy gap $\Delta(t)$ caused by the fluctuation of the electric field would lead to unwanted phase to the desired gate operations.

We use the two-qubit gate in Eq. (9) as an example. With the time dependent fluctuations $\delta \lambda(t)$ of the effective coupling coefficient $\lambda=g^{2} / \delta$, the evolution operator of the system becomes $U_{2 q}^{\prime}=$ $U_{2 q} \exp \left\{-\mathrm{i} \int_{0}^{t_{2 q}} \mathrm{~d} t \delta \lambda(t)\left(\frac{3}{2} \sum_{i=1,2} \hat{\sigma}_{z}^{i}+\hat{\sigma}_{+}^{1} \hat{\sigma}_{-}^{2}+\hat{\sigma}_{-}^{1} \hat{\sigma}_{+}^{2}\right)\right\}$, where the unwanted phase $\phi=\int_{0}^{t_{2 q}} \mathrm{~d} t \delta \lambda(t)$. The distribution of the unwanted phase becomes Gaussian distribution because $\lambda$ is in Gaussian distribution. With the parameters above, we numerically calculate the variances of the unwanted phase $\operatorname{Var}(\phi) \sim 5 \times 10^{-3} \pi$.

For single-qubit $\sigma_{x}$ gate, the unwanted phase is $\int_{0}^{t_{x}} \mathrm{~d} t \delta \Omega_{\mathrm{R}}(t)$, while for $\sigma_{z}$ gate, that becomes $\int_{0}^{t_{z}} \mathrm{~d} t \delta \Omega_{\mathrm{R}}^{\prime}(t)$. With the same method, we can calculate the variance of the phases.

From the analysis, we show that even the dephasing occurs over the gate operation, we can still implement a universal set of gates with high fidelities. For example, with the parameters we show above the fidelity for the entangling two-qubit gate is about $0.9946,0.9952$ for $\sigma_{x}$ gate and 0.9961 for $\sigma_{z}$ gate.

\section{SUMMARY}

If a quantum computer is built, intractable problems such as factorization would be solved efficiently, with enormous ramifications for communication security. Semiconductor DDMs quantum computer, which would capitalize on chip fabrication technology and could be hybridized with existing computers, is the preferred method for quantum computation. Here we propose scalable quantum computing with electrically controlled semiconductor spins of DDMs coupled to a microwave stripline resonator on a chip. Quantum information is encoded in the singlet states of DDMs. Initialization of qubits can be realized with an adiabatic passage. With the switchable coupling to the resonator, we can implement a universal 
set of quantum gates on any qubit. Although in general charge qubits have less coherent life time compared to spin qubits, the generation and measurement methods are much simpler and faster, which makes our protocol competitive with spin qubits in the context of circuitbased quantum computing. Because of the switchable coupling between the double-dot pairs and the resonator, we can apply this entangling gate on any two qubits without affecting others, which is not trivial for implementing scalable quantum computing and generating large entangled state. The fidelities of the gates in our protocol are studied including all kinds of major decoherence, with promising results for reasonably achievable experimental parameters. The feasibility of this scheme is char- acterized through exact numerical simulations that incorporate various sources of experiment noise and these results demonstrate the practicality by way of current experimental technologies.

\section{ACKNOWLEDGEMENTS}

This work has been supported by National Natural Science Foundation of China, Grant No. 10944005, Southeast University Startup fund, NSERC, MITACS, CIFAR, QuantumWorks and iCORE.
[1] L. Grover, Phys. Rev. Lett. 79 (1997) 325-328.

[2] P.W. Shor, Proc. 35th Annual Symp. on Found. of Comp. Sci. (Los Alamitos, CA: IEEE Computer Society Press, 1994) 124-130.

[3] D. Loss, D.P. DiVincenzo, Phys. Rev. A 57 (1998) 120126.

[4] B.E. Kane, Nature 393 (1998) 133-137.

[5] S.E.S. Andresen et al., Nanolett. 7 (2007) 2000-2003.

[6] A. Wallraff et al., Phys. Rev. Lett. 95 (2005) 060501.

[7] A. Imamoglu et al., Phys. Rev. Lett. 83 (1999) 4204-4207.

[8] J.R. Petta et al., Science 309 (2005) 2180-2184.

[9] A.C. Johnson, Nature 435 (2005) 925-928.

[10] G. Burkard, A. Imamoglu, Phys. Rev. B 74 (2006) 041307(R).

[11] J.M. Taylor et al., Phys. Rev. B 76 (2007) 035315.
[12] J.M. Taylor, M.D. Lukin, arXiv: cond-mat/0605144

[13] Z.R. Lin et al., Phys. Rev. Lett. 101 (2008) 230501.

[14] D.P. DiVincenzo, Fortschr. Phys. 48 (2000) 771-783.

[15] A. Blais et al., Phys. Rev. A 75 (2007) 032329.

[16] S.B. Zheng, Phys. Rev. A 66 (2002) 060303(R).

[17] A. Blais et al., Phys. Rev. A 69 (2004) 062320.

[18] T. Hayashi et al., Phys. Rev. Lett. 91 (2003) 226804; J.R. Petta et al., ibid 93 (2004) 186802.

[19] A. Wallraff et al., Nature 431 (2004) 162-167.

[20] L. Frunzio et al., Applied Superconductivity, IEEE Transactions on 15 (2005) 860-867.

[21] M.B. Haider et al., Phys. Rev. Lett. 102 (2009) 046805; L. Livadaru, P. Xue et al., arXiv: 0910.1797. 